\title{
Hyperfine Suppression of $2{ }^{3} \mathrm{~S}_{1}-3{ }^{3} \mathrm{P}_{J}$ Transitions in ${ }^{3} \mathrm{He}$
}

\author{
I. A. Sulai,,${ }^{1,2,3}$ Qixue Wu, ${ }^{4}$ M. Bishof, ${ }^{1,3}$ G. W. F. Drake, ${ }^{4}$ Z.-T. Lu, ${ }^{1,2,3}$ P. Mueller, ${ }^{1}$ and R. Santra ${ }^{2,5}$ \\ ${ }^{1}$ Physics Division, Argonne National Laboratory, Argonne, Illinois 60439, USA \\ ${ }^{2}$ Department of Physics, The University of Chicago, Chicago, Illinois 60637, USA \\ ${ }^{3}$ The Enrico Fermi Institute, The University of Chicago, Chicago, Illinois 60637, USA \\ ${ }^{4}$ Department of Physics, University of Windsor, Windsor, ON N9B 3P4, Canada \\ ${ }^{5}$ Chemical Sciences and Engineering Division, Argonne National Laboratory, Argonne, Illinois 60439, USA
}

(Dated: November 4, 2018)

\begin{abstract}
Two anomalously weak transitions within the $2{ }^{3} \mathrm{~S}_{1}-3{ }^{3} \mathrm{P}_{J}$ manifolds in ${ }^{3} \mathrm{He}$ have been identified. Their transition strengths are measured to be 1,000 times weaker than that of the strongest transition in the same group. This dramatic suppression of transition strengths is due to the dominance of the hyperfine interaction over the fine structure interaction. An alternative selection rule based on $I S$-coupling (where the nuclear spin is first coupled to the total electron spin) is proposed. This provides qualitative understanding of the transition strengths. It is shown that the small deviations from the $I S$-coupling model are fully accounted for by an exact diagonalization of the strongly interacting states.
\end{abstract}

PACS numbers: 31.30.Gs, 32.10.Fn, 32.70.-n, 32.70.Fw, 32.70.Cs

Persistent efforts in both theory and experiment have yielded increasingly precise understanding of the helium atom. Due to its simplicity, the helium atom has been a proving ground for precision atomic measurements and calculations of few-body quantum systems. The knowledge gained from this effort is used to test bound-state quantum electrodynamics [1, 2, 3], determine the fine structure constant [4, 5], and explore exotic nuclear structure [6, 7, 8, 9]. We report results of a combined theoretical and experimental study on the strengths of $2{ }^{3} \mathrm{~S}_{1}-3{ }^{3} \mathrm{P} J$ transitions in ${ }^{3} \mathrm{He}$.

Surprisingly, we observe that the strengths of two "allowed" transitions, $2{ }^{3} \mathrm{~S}_{1},\left(F=\frac{3}{2}\right)-3{ }^{3} \mathrm{P}_{1},\left(F=\frac{3}{2}\right)$ and $2{ }^{3} \mathrm{~S}_{1},\left(F=\frac{1}{2}\right)-3{ }^{3} \mathrm{P}_{2},\left(F=\frac{3}{2}\right)$, are 1,000 times weaker than that of the strongest transition $2{ }^{3} \mathrm{~S}_{1},(F=$ $\left.\frac{3}{2}\right)-3{ }^{3} \mathrm{P}_{2},\left(F=\frac{5}{2}\right)$. The level scheme showing these transitions is presented in Fig 1. This dramatic suppression of transition strengths is due to a rare atomic phenomenon: within the $3{ }^{3} \mathrm{P}$ manifold, the hyperfine interaction is comparable to or even stronger than the fine structure interaction. Consequently the conventional model based on $L S$-coupling is no longer applicable. Rather, we find that an alternative model where the fine structure interaction is treated as a perturbation on states obtained by first coupling nuclear spin to the total electron spin provides a good qualitative explanation of the observed suppression. We refer to this coupling scheme as $I S$-coupling. We start by discussing the details of the experiment and compare the data with the predictions from the different coupling schemes. Finally, we discuss an exact diagonalization method to account for the small differences between experiment and the $I S$ coupling scheme.

We measure the ratio of transition strengths using a cross-beam laser induced fluorescence method. A beam of metastable helium atoms in the $2{ }^{3} \mathrm{~S}_{1}$ state is prepared in a liquid-nitrogen cooled RF-driven discharge. A retro-reflected beam of linearly polarized $389 \mathrm{~nm}$ light is

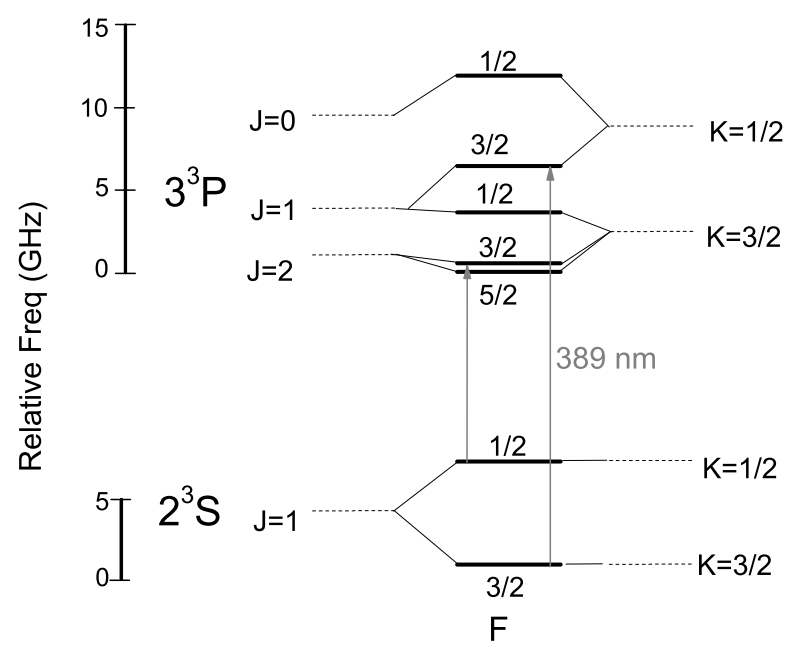

FIG. 1: Level scheme of ${ }^{3} \mathrm{He}$ showing the levels investigated, with the arrows indicating the suppressed transitions observed. The level positions are drawn to scale within each manifold. The large hyperfine splitting with respect to the fine structure splitting is evident. With a nuclear spin $I=1 / 2$ for ${ }^{3} \mathrm{He}$, the levels are designated by the familiar term symbols, with $\mathbf{J}=\mathbf{L}+\mathbf{S}, \mathbf{F}=\mathbf{J}+\mathbf{I}$ on the left. The levels are labeled on the right using the quantum number $\mathbf{K}=\mathbf{I}+\mathbf{S}$, $\mathbf{F}=\mathbf{K}+\mathbf{L}$.

incident perpendicular to the atomic beam. The polarization of the light is along the direction of the atomic beam. A uniform external magnetic field of 5 Gauss is applied along the direction of the laser to provide an axis of quantization. As the frequency is scanned across different resonances, the atoms are excited, and fluorescence from the atoms is detected in the direction normal to the atomic and laser beams. The metastable atomic beam is collimated using a collimator, made of a stack of microscope cover slips which provides high collimation in the direction along the laser beam [10]. We are able to ob- 
tain Doppler broadened lines of $20 \mathrm{MHz}$ linewidth. The natural linewidth of the transitions is $1.6 \mathrm{MHz}$. Approximately $4 \mathrm{~mW}$ of $389 \mathrm{~nm}$ light is obtained by frequency doubling infrared light at $778 \mathrm{~nm}$. The frequency of the $778 \mathrm{~nm}$ light is referenced to a temperature stabilized Fabry-Perot cavity. The power of the laser and its wavelength are monitored continuously.

The nine E1 allowed transitions are repeatedly probed in a random order and the spectra are recorded. Each spectrum is fitted using a statistically weighted Voigt profile. The integrated area of the profile divided by the power of the probing laser beams is taken as a measure of the transition strength. As the absolute atomic beam flux and efficiency of detecting the fluorescence photons are not measured in this experiment, only the ratios of transition strengths are determined. By defining the strength of the strongest transition, $2{ }^{3} \mathrm{~S}_{1},\left(F=\frac{3}{2}\right)-3{ }^{3} \mathrm{P}_{2},(F=$ $\left.\frac{5}{2}\right)$, to be unity, we determine the relative strengths of the other eight transitions. The results are presented in Fig. 2 and in Table 1 .

The intensity of the probing laser beam is varied depending on the transition under study. For example, when probing the two highly suppressed transitions, the intensity of the probe is increased by two orders of magnitude. In all cases, however, the laser intensity is kept well below the saturation intensity of the particular transition under study. Indeed, the intensity is chosen so that on average less than one photon is scattered by each atom as it passes the laser beams in approximately $2 \mu \mathrm{s}$. This is to avoid nonlinear effects in the measurements due to optical pumping and mechanical effects of the light on the atomic beam. Such systematic effects are studied by examining the dependence of transition signal on the laser beam power. Additional corrections are made and systematic errors generated due to changing background in the measured laser power and the anisotropic angular distribution of the fluorescence emission. The final error estimates are given in Table $\llbracket$.

TABLE I: Relative transition strengths for all E1 allowed transitions between the $2{ }^{3} \mathrm{~S}_{1}$ and $3{ }^{3} \mathrm{P}_{J}$ manifolds. All values are normalized with respect to the $2{ }^{3} \mathrm{~S}_{1},\left(F=\frac{3}{2}\right)-$ $3{ }^{3} \mathrm{P}_{2},\left(F=\frac{5}{2}\right)$ transition.

\begin{tabular}{ccllll}
\hline \hline $\begin{array}{c}\text { Initial }(\mathrm{J}, \mathrm{F}) \\
2{ }^{3} \mathrm{~S}_{J}\end{array}$ & $\begin{array}{c}\text { Final }(\mathrm{J}, \mathrm{F}) \\
3{ }^{3} \mathrm{P}_{J}\end{array}$ & Experiment & LS & IS & $\begin{array}{l}\text { Exact } \\
\text { Diag. }\end{array}$ \\
\hline \multirow{3}{*}{$(1,3 / 2)$} & $(2,5 / 2)$ & 1 & 1 & 1 & 1 \\
& $(2,3 / 2)$ & $0.69(5)$ & 0.11 & 0.67 & 0.67 \\
& $(1,1 / 2)$ & $0.26(4)$ & 0.11 & 0.33 & 0.24 \\
& $(1,3 / 2)$ & $0.0012(2)$ & 0.55 & 0 & 0.0010 \\
& $(0,1 / 2)$ & $0.10(5)$ & 0.22 & 0 & 0.093 \\
\hline \multirow{3}{*}{$(1,1 / 2)$} & $(2,3 / 2)$ & $0.0011(4)$ & 0.55 & 0 & 0.0010 \\
& $(1,1 / 2)$ & $0.08(3)$ & 0.22 & 0 & 0.093 \\
& $(1,3 / 2)$ & $0.65(4)$ & 0.11 & 0.67 & 0.67 \\
\hline \hline
\end{tabular}

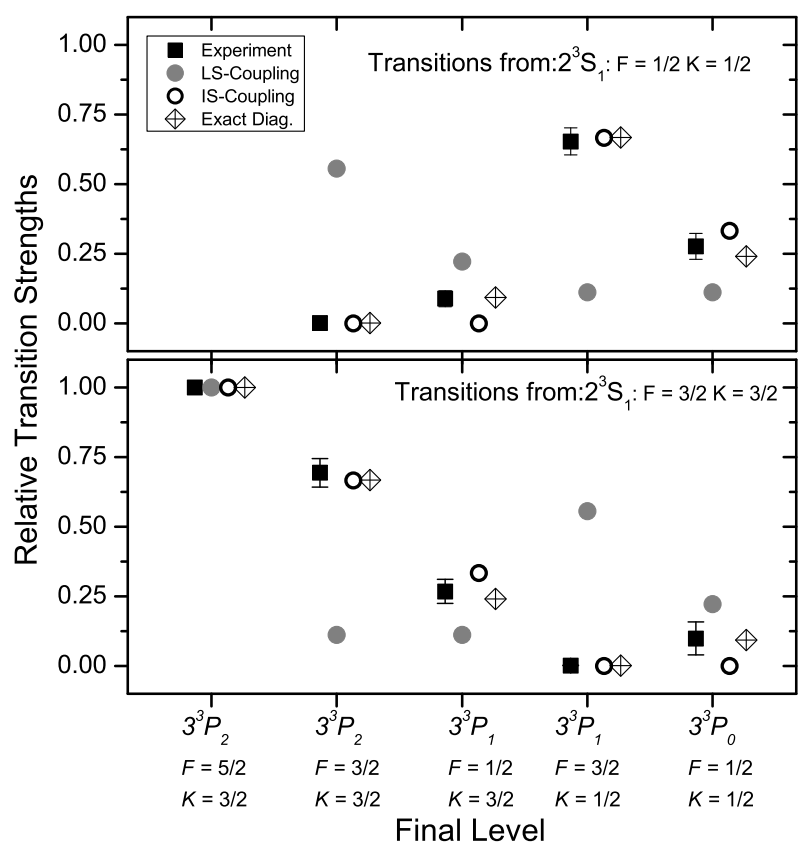

FIG. 2: Comparison of relative transition strengths for all E1 allowed transitions between the $2{ }^{3} \mathrm{~S}_{1}$ and $3{ }^{3} \mathrm{P}_{J}$ manifolds. All values are normalized with respect to the $2{ }^{3} \mathrm{~S}_{1},(F=$ $\left.\frac{3}{2}\right)-3{ }^{3} \mathrm{P}_{2},\left(F=\frac{5}{2}\right)$ transition.

The textbook strategy [1] to estimate theoretically the atomic transition strengths is based on the presumed hierarchy that hyperfine splittings be small in comparison with fine-structure splittings. Consequently approximate eigenstates of the total Hamiltonian may be constructed by first coupling $L$ (total orbital angular momentum quantum number) and $S$ (total electronic spin quantum number) to form the total electronic angular momentum $J$; coupling $J$ and $I$ (nuclear spin quantum number) then gives the total atomic angular momentum $F$. Within this $L S$-coupling model, the total strength for an electric dipole transition may be evaluated using standard angular momentum algebra [12].

The results of this $L S$-coupling model are compared with the experimental data in Fig. 2. It is apparent that there is not even qualitative agreement. The origin of the failure of the $L S$-coupling model may be understood as follows. In ${ }^{3} \mathrm{He}$, the hyperfine structure is almost entirely due to the magnetic dipole interaction of the tightly bound $1 s$ electron with the nucleus. The fine structure is a consequence of both one-body spin-orbit coupling of the excited $n L$ electron and two-body spin-other-orbit and spin-spin interactions of the $n L$ electron with the $1 s$ electron [13]. As $n$ increases, the fine-structure splittings decrease as $n^{-3}$. The hyperfine interaction of the $1 s$ electron, on the other hand, tends for large $n$ to the constant hyperfine interaction strength in ${ }^{3} \mathrm{He}^{+}$. Note that the hyperfine splitting in the ground state of ${ }^{3} \mathrm{He}^{+}$ is $8.7 \mathrm{GHz}$ 14], which is comparable to, or larger than, the level spacings within the $2{ }^{3} S$ and $3{ }^{3} P$ manifolds 
(see Fig. 1).

The relative strength of the hyperfine interaction in ${ }^{3} \mathrm{He}$ has been recognized before [15, 16, 17, 18, 19, 20, 21] and has been taken as an indication that a simple angular momentum coupling model describing transitions in ${ }^{3} \mathrm{He}$ is not available and that a numerical diagonalization of an effective Hamiltonian is necessary. We demonstrate in the following that although $n$ is quite small in the $3{ }^{3} \mathrm{P}$ manifold, the assumption of relatively weak fine structure interactions does provide a simple model that allows us to understand qualitatively the strengths of transitions from $2{ }^{3} \mathrm{~S}$ to $3{ }^{3} \mathrm{P}$.

For $3{ }^{3} \mathrm{P}, \mathrm{S}$ is still a good quantum number, since the separation of this manifold from $3^{1} \mathrm{P}$ is large $(\sim$ $10^{4} \mathrm{GHz}$ ) in comparison with the hyperfine and fine structure splittings. Therefore, the basic idea underlying what we refer to as the $I S$-coupling model is that the electrostatic exchange interaction between the two electrons preserves $\mathbf{S}$; the hyperfine interaction couples $\mathbf{S}$ and $\mathbf{I}$ to form a new intermediate angular momentum $\mathbf{K}$; and $\mathbf{F}$ is then obtained by coupling $\mathbf{L}$ and $\mathbf{K}$. In this picture, the ${ }^{3} \mathrm{He}$ eigenstates of relevance here are not labeled in terms of $n L S(J) I, F$, but in terms of $n I S(K) L, F$. An immediate consequence of the fact that the electric dipole operator acts on neither $S$ nor $I$ is that $K$ must be conserved in an $E 1$ transition, i.e., $\left|\left\langle\Psi_{K^{\prime} F^{\prime}}^{\left(n^{\prime} L^{\prime} I\right)}\|\hat{D}\| \Psi_{K F}^{(n L S I)}\right\rangle\right|^{2}$ vanishes if $K$ differs from $K^{\prime}$. A similar model was used in 1933 for a case in which $S$ is not conserved [22], but that appears to be the only other study employing an extreme hyperfine-coupling picture to develop a basic understanding of transition strengths involving hyperfine multiplets.

As shown in Fig. 2, there is good qualitative agreement between experiment and the IS-coupling model, thus suggesting that already for $n=3$, the fine-structure interactions may be considered perturbations to the hyperfine structure. For instance, within the $I S$-coupling model, the suppression of the transition from $2{ }^{3} \mathrm{~S}_{1}$, $F=\frac{3}{2}\left(K=\frac{3}{2}\right)$ to $3{ }^{3} \mathrm{P}_{1}, F=\frac{3}{2}\left(K=\frac{1}{2}\right)$ follows from the $K$-selection rule in $E 1$ transitions. On the other hand, according to experiment, the transition from $2{ }^{3} \mathrm{~S}_{1}$, $F=\frac{3}{2}\left(K=\frac{3}{2}\right)$ to $3{ }^{3} \mathrm{P}_{0}, F=\frac{1}{2}\left(K=\frac{1}{2}\right)$ is weakly allowed, in slight deviation from the $I S$-coupling model. We note that the observed suppressions for certain transitions from $2{ }^{3} \mathrm{~S}$ to $5{ }^{3} \mathrm{P}[19,21]$ are fully consistent with the $K$-selection rule.

In order to characterize the nature of the perturbations to the $I S$-coupling model for ${ }^{3} \mathrm{He}$, and account for the slight deviations, we have performed an exact diagonalization of the total Hamiltonian $H$ within the manifold of $3{ }^{3} \mathrm{P}$ and $3{ }^{1} \mathrm{P}$ states, including both fine and hyperfine structure. The total Hamiltonian of ${ }^{3} \mathrm{He}$ in the absence of external fields is of the form

$$
H=H_{\mathrm{NR}}+H_{\mathrm{fs}}+H_{\mathrm{hfs}}
$$

where $H_{\mathrm{NR}}$ is the nonrelativistic Hamiltonian, $H_{\mathrm{fs}}$ represents the fine structure interaction for helium as described by many authors (see Drake [23, 24] for a review), and $H_{\mathrm{hfs}}$ represents the hyperfine structure interaction, see for example, Bethe and Salpeter [13]. In this picture, $H_{\mathrm{hfs}}$ is treated as a small perturbation relative to the large electrostatic splitting between states with different principal quantum number $n$, and by exact diagonalization within the manifold of strongly mixed states with the same $n$. The technique is basically the same as that described by Hinds, Prestage and Pichanick [25].

Using these methods, a comprehensive investigation of the fine and hyperfine structure of ${ }^{3} \mathrm{He}$ has recently been carried out by Morton, Wu, and Drake 2]. All fine structure and hyperfine structure parameters required to diagonalize the complete fine and hyperfine interaction matrix in the basis set of singlet and triplet states are accurately calculated by using double basis set variational wave functions in Hylleraas coordinates as described by Drake 23, 24]. For the $3 P$ state, instead of using directly the theoretical energies for ${ }^{3} \mathrm{He}$, we combined the theoretical isotope shifts for ${ }^{3} \mathrm{He}$ relative to ${ }^{4} \mathrm{He} 2$ 2] with the best experimental ionization energies for ${ }^{4} \mathrm{He} \cdot[2,7]$. This gives higher accuracy due to cancelations of the massindependent QED uncertainties in the calculated isotope shifts.

The final step is to calculate the electric dipole transition line strengths between the perturbed hyperfine states of $2{ }^{3} \mathrm{~S}$ and $3{ }^{3} \mathrm{P}$ in terms of standard angular momentum theory, in which the perturbed hyperfine states are linearly expanded in terms of unperturbed eigenstates. The expansion coefficients are obtained by the above diagonalization of the complete matrix. The final results and the comparison with experiment are given in Table [. The calculations show that the mixing between hyperfine states of $3{ }^{3} \mathrm{P}$ with different $K$ but the same $F$ of ${ }^{3} \mathrm{He}$ precisely accounts for the deviations shown in Table 【 from the $I S$-coupling model. This mixing is due to the fine structure interactions. We find that both the one-body spin-orbit, and the two-body spin-spin and spin-other-orbit interactions must be included, in order to accurately reproduce the strengths. In the case of the $2 S$ state, this hyperfine mixing is also important for hyperfine structure, as shown by Riis et al. [26], but its contribution to the transition strength is negligible in the present work.

In summary, the hyperfine suppression of $2{ }^{3} \mathrm{~S}_{1},(F=$ $\left.\frac{3}{2}\right)$ to $3{ }^{3} \mathrm{P}_{1},\left(F=\frac{3}{2}\right)$ and $2{ }^{3} \mathrm{~S}_{1},\left(F=\frac{1}{2}\right)$ to $3{ }^{3} \mathrm{P}_{2},(F=$ $\left.\frac{3}{2}\right)$ radiative transitions in ${ }^{3} \mathrm{He}$ is caused by a selection rule that emerges in the limit of strong hyperfine mixing between states with the same $F$ but different $J$. In this limit, the radiative transitions are better described by a coupling scheme in which $\mathbf{I}$ and $\mathbf{S}$ are coupled to form $\mathbf{K}$, and then $\mathbf{L}$ is coupled to $\mathbf{K}$ to form $\mathbf{F}$. In this limit, the eigenvalue $K$ is approximately preserved as a good quantum number. The small deviations from the $I S$-coupling scheme are well accounted for by an exact diagonalization for the intermediate coupling case. However, with increasing $n$, the $I S$-coupling scheme should rapidly become more accurate because the fine-structure interactions decrease in proportion to $1 / n^{3}$, while the hyperfine interactions tend to a constant at the series limit. 
The surprise is that it already works so well for $n=3$.

We would like to thank K. Bailey and T. P. O'Connor for technical support. This work was supported by the U.S. Department of Energy, Office of Nuclear Physics and
Office of Basic Energy Sciences, Office of Science, under Contract No. DE-AC02-06CH11357. G.W.F.D. acknowledges support by the Natural Sciences and Engineering Research Council of Canada, and by SHARCNET.
[1] D. Morton, Q. Wu, and G. W. F. Drake, Phys. Rev. A 73, 034502 (2006).

[2] D. Morton, Q. Wu, and G. W. F. Drake, Can. J. Phys. 84, 83 (2006).

[3] K. Pachucki and J. Sapirstein, Journal of Physics B: Atomic, Molecular and Optical Physics 36, 803 (2003).

[4] T. Zelevinsky, D. Farkas, and G. Gabrielse, Phys. Rev. Lett 95, 203001 (2005).

[5] M. C. George, L. D. Lombardi, and E. A. Hessels, Phys. Rev. Lett. 87, 173002 (2001).

[6] L.-B. Wang, P. Mueller, G. W. F. Drake, J. P. Greene, D. Henderson, R. J. Holt, R. V. F. Janssens, C. L. Jiang, Z.-T. Lu, T. P. O'Connor, et al., Phys. Rev. Lett 93, 142501 (2004).

[7] P. Mueller, L.-B. Wang, G. W. F. Drake, K. Bailey, Z.T. Lu, and T. P. O'Connor, Phys. Rev. Lett 94, 133001 (2005).

[8] D. Shiner, R. Dixson, and V. Vedantham, Phys. Rev. Lett 74, 3553 (1995).

[9] F. Marin, F. Minardi, F. S. Pavone, M. Inguscio, and G. W. F. Drake, Z. Phys. D 32, 285 (1995).

[10] V. Gerginov and C. E. Tanner, Optics Communications 222, 17 (2003).

[11] I. I. Sobelman, Atomic Spectra and Radiative Transitions (Springer, Berlin, 1979).

[12] A. R. Edmonds, Angular Momentum in Quantum Mechanics (Princeton University Press, Princeton, New Jersey, 1996).

[13] H. A. Bethe and E. E. Salpeter, Quantum Mechanics of One- and Two-Electron Atoms (Springer, Berlin, 1957).
[14] E. N. Fortson, F. G. Major, and H. G. Dehmelt, Phys. Rev. Lett. 16 (1996).

[15] M. Fred, F. S. Tomkins, J. K. Brody, and M. Hamermesh, Phys. Rev. 82, 406 (1951).

[16] K. Tillmann, H. J. Andra, and W. Wittmann, Phys. Rev. Lett. 30, 155 (1973).

[17] J. Derouard, M. Lombardi, and R. Jost, J. Phys. France 41, 819 (1980).

[18] R. R. Freeman, P. F. Liao, R. Panock, and L. M. Humphrey, Phys. Rev. A 22, 1510 (1980).

[19] L. A. Bloomfield, B. Couillaud, P. Dabkiewicz, H. Gerhardt, and T. W. Haensch, Phys. Rev. A 26, 713(R) (1982).

[20] J. D. Prestage, E. A. Hinds, and F. M. J. Pichanick, Phys. Rev. Lett. 50, 828 (1983).

[21] W. Vassen and W. Hogervorst, Phys. Rev. A 39, 4615 (1989).

[22] S. Goudsmit and R. F. Bacher, Phys. Rev. 43, 894 (1933).

[23] G. W. F. Drake, in Long-Range Casimir Forces:Theory and Recent Experiments in Atomic Systems., edited by F. S. Levin and D. A. Micha (Plenum, New York, 1993).

[24] G. W. F. Drake, in Atomic, Molecular, and Optical Physics Handbook, edited by G. W. F. Drake (Springer, New York, 2006).

[25] E. Hinds, J. Prestage, and F. Pichanick, Phys. Rev. A 33, 68 (1986).

[26] E. Riis, A. Sinclair, O. Poulsen, G. Drake, W. Rowley, and A. Levick, Phys. Rev. A 49, 207 (1994). 